is, from the time the call was made) from those transported by the routine ambulance service. The predictable difference in overall mortality has never been used as evidence of the salutary effect of prehospital coronary care. Results obtained with the Belfast mobile coronary care unit (MCCU) indicate that the outlook of patients seen and treated early (mortality $\left.10^{\circ}{ }_{0}\right)^{2}$ is better than that of patients admitted to hospital coronary care units at a time of lower risk by traditional means (hospital mortality $15-20^{\circ}$ ) and much better than that of patients seen early but not given intensive care (mortality $26 \%$ ). ${ }^{3}$

Of course the existence of an MCCU by itself cannot prevent many of those deaths which occur within the first minutes of an attack. Where an MCCU is available, however, much more can be done, through the establishment of "satellite" units in densely populated factories and office blocks ${ }^{4}$ and the training of large numbers of paramedical personnel in the technique of resuscitation, ${ }^{5}$ to prevent early death from ventricular fibrillation.

Dr Hampton found that more lives might have been saved had the "cardiac ambulance" been dispatched more often while it was available. The criteria for selection of calls from the public are crucial. With a short response time for the special ambulance we would question the logic of sending a routine ambulance following a 999 call if it was nearer the patient. Dr Hampton's report does not question the unassailable fact that the principal causes of death-ventricular fibrillation and extension of the infarct due to autonomic disturbance-are mainly operative outside hospital. Since the means of preventing death are available and relatively inexpensive the logic of providing prehospital coronary care is irrefutable. Much the same argument could be used to justify the continuation of the fire service were it under attack.

Direct measurement of the impact on community mortality cannot be made entirely from the results of operation of an MCCU. Nevertheless, an estimate can be made, ${ }^{6}$ and a decline in community mortality coinciding with the inception of an MCCU has been observed.?

J S GEDDES Royal Victoria Hospital,

A A J ADGEY

Belfast

Working Party of the Royal College of Physicians and the British Cardiac Society, Journa

2 Pantridge, J F, Quarterly fournal of Medicine, 1970

39, 621.
Mather, H G, et al, British Medical fournal, 1971, 3, 334.

Grace, W J, in Coronary Care: Pre-hospital Care of
Acute Myocardial Infarction. New York, American Heart Association, 1973.

${ }^{5}$ Cobb, L A, and Alvarez, H, III, National Conference on Standards for Cardiopulmonary Resuscitation $(C P R)$ and Emergency Cardiac Care $(E C C)$. Washington, DC, National Research

- Pantridge, J F, et al, The Acute Coronary Attack. Tunbridge Wells, Pitman Medical, 1975.

Crampton, R S, et al, American Journal of Medicine, $1975,58,151$.

\section{Sigmoid volvulus in Africa}

SIR,-The article by Mr H G Sturzaker and others (8 November, p 338) about recurrent sigmoid volvulus touched on a subject of interest to readers in Africa, where this condition is a frequent cause of lower intestinal obstruction. It is generally accepted that two different varieties of sigmoid volvulus occur here, an acute fulminating and a slowly pro- gressive, subacute form, each with its own specific clinical and pathological characteristics. ${ }^{1}$

The case histories reported by Mr Sturzaker and his colleagues suggest that all their patients suffered from the subacute type, but other findings are not consistent with it. Most of the attacks described ended spontaneously, while all patients here need at least decompression with a flatus tube passed through a sigmoidoscope, if not a laparotomy. Also the sex ratio is the reverse of that seen in Britain. Subacute volvulus is even rare in women; if the patient presenting with a volvulus is a woman this is a strong argument against attempting conservative treatment." And last of all, the gross pathology is quite different from that shown in the photograph accompanying the article. Two cases with muscular hypertrophy were found in the series reported, whereas subacute cases in Africa, besides the white striae of the mesocolon, invariably show loss of haustrations and taeniae spread out as a continuous muscular coat.

Because of these differences it remains an open question whether we have to do with the same disease.

Mpwapwa District Hospital,

J J MeuzelaAR Mpwapwa,

'Davey, W W, Companion to Surgery in Africa, p 235. Shepherd, J J, Tropical Doctor, 1971, 1, 174.

\section{Infections after splenectomy in Hodgkin's} disease

SIR,-The paper by Dr B W Hancock and others (7 February, p 313) draws attention to the severe infection which may occasionally follow splenectomy. King and Shumaker first reported on this in 1952, ${ }^{1}$ and several groups have confirmed their findings since then. The pattern of abrupt onset and a rapid fulminating course is borne out by the experience of $\mathrm{Dr}$ Hancock and his colleagues, but the usual infection with pneumococci, Neisseria meningitidis, or Haemophilus influenzae was seen in only one of their patients.

The reason for the increased susceptibility to infection in splenectomised patients is not known. A fall in IgM levels following splenectomy has been described previously. ${ }^{2}$ Other immunoglobulins are unaffected. The antibody response in splenectomised patients to immunisation subcutaneously is normal, ${ }^{3}$ but with intravenous immunisation ${ }^{4}$ it is feeble and delayed. This may play a role in their liability to septicaemia. Little evidence is provided by Dr Hancock and his colleagues that cell-mediated immunity is affected by splenectomy, and in a previous study none was found. ${ }^{8}$

The susceptibility to infection in such patients with Hodgkin's disease will be complicated by the immunosuppressive and myelosuppressive effects of treatment by radiotherapy or cytotoxic drugs and the effect of the disease itself. It has been suggested, and is our experience to date, that splenectomised patients with Hodgkin's disease are better able to tolerate radiotherapy ${ }^{6}$ and cytotoxic therapy. ${ }^{7}$ Blood leucocyte and platelet counts may give a false sense of security in these patients, and greater bone marrow damage could follow their more vigorous therapy. The patients of Dr Hancock and his colleagues were reported to be leucopenic, but we are not told how vigorous a leucocytosis they were able to mount in the face of their overwhelming infection or indeed the severity of the leucopenia.

The crux of their paper is whether the increased susceptibility to infection justifies splenectomy in patients with Hodgkin's disease. To make a judgment we have to know what risks are involved. The value of splenectomy in detecting disease in the spleen and facilitating comprehensive therapy is established. ${ }^{8} 9$ The underlying disease influences the incidence of infection in splenectomised patients. In Hodgkin's disease the incidence of serious infection has been reported at $1.4 \%$ with a mortality of $0.5 \%$ from a survey of 1170 splenectomised patients. ${ }^{10}$ Surprisingly, this is a lower incidence than seen in patients splenectomised for nonmalignant conditions. Predisposing factors in reported cases are youth and exposure to quadruple chemotherapy.

The improved survival with better staging and treatment probably outweighs the risk of infection. When improved techniques for detecting and treating splenic disease are available the problem will be resolved. In the meantime it is necessary to identify groups in whom it is safe not to perform splenectomy and to take into account the special susceptibility of children. As severe infection occurs predominantly within two years of splenectomy careful surveillance is called for, particularly as most patients will be receiving radiotherapy or cytotoxic drugs during that time. It has also been suggested that children should be given penicillin cover during the period at risk. ${ }^{11}$

P AMLot

J R TROUNCE

Medical Oncology Unit,

Guy's Hospital,

London SE

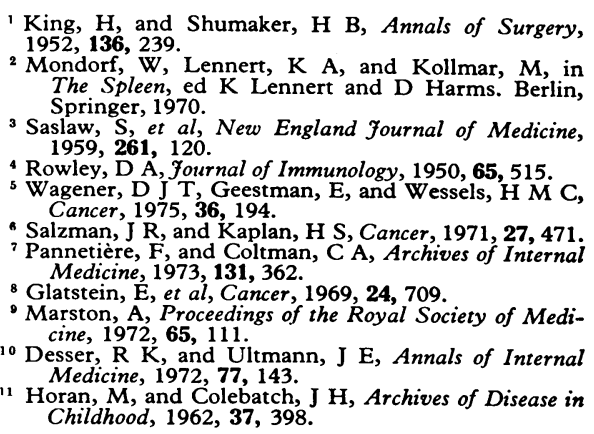

Childhood, 1962, 37, 398 .

\section{Selective induction of labour}

SIR,-Dr P W Howie and his colleagues (17 January, $p$ 150) express doubt as to the validity of the results of Professor K O'Driscoll's prospective study of 1000 primigravidae in which the induction rate was $9.5 \%$ and caesarean section rate $5 \cdot 1 \%$. The Glasgow group question if any disadvantages arise from induction of labour and infer that such a policy will result in a reduction of caesarean sections. This is a surprising statement when in their own study ${ }^{1}$ of elective induction in 228 healthy pregnant women selected at 38 weeks' gestation $67.5 \%$ of the total were induced and $6.1 \%$ required caesarean section. This is a high caesarean section rate for such patients and Dr Howie might like to compare the annual rate of induction and incidence of operative deliveries at the Glasgow Royal 